Artículo

\title{
Actividad reproductiva y longitud de madurez de sardina austral Sprattus fuegensis en el mar interior de Chiloé, Chile
}

\author{
Reproductive activity and maturity length of Patagonian sprat
} Sprattus fuegensis in Chiloé inland seawaters, Chile

\section{Elson Leal ${ }^{1}$, T. Mariella Canales ${ }^{1,2}$, Antonio Aranis ${ }^{1}$ y Margarita Gonzáles ${ }^{1}$}

\author{
${ }^{1}$ Instituto de Fomento Pesquero, Blanco 839, Valparaíso, Chile. elson.leal@ifop.cl
}

${ }^{2}$ University of York, Department of Biology, York, YO10 5DD, United Kingdom

\begin{abstract}
The Patagonian sprat, Sprattus fuegensis, has been recently identified in the catches of small pelagic fish in the inland waters of Chiloé, southern Chile. In spite of its increasing economic importance, there are still unknown aspects of its reproductive biology. This study describes the reproductive activity of females of this species based on monthly variation of the Gonadosomatic Index (IGS) and the frequency of macroscopic states of maturity between J anuary of 2006 and December of 2009 and microscopic states since February of 2007. The maturity at length of females was studied during the main spawning season. IGS monthly variation and the frequency of macroscopic states of maturity reached maximum values each year, between September and October, and minimum from J anuary to J une. In addition, the histological analysis of the ovaries confirmed that $\mathrm{S}$. fueguensis is a partial spawner whose reproductive activity although tends to concentrate between September and October can be extended to December. The mean length at maturity of females during the reproductive season was $13.5 \mathrm{~cm}$, indicating that they spawned at longer lengths in comparison with the same species from other localities. It is discussed that the increase of the reproductive activity in spring and larger maturity sizes could be part of a reproductive strategy of the species to enhance the survival during early stages in the inland seawater of Chiloé.
\end{abstract}

Key words: Reproduction, spawning season, reproductive strategy, pelagic fish

Resumen. - La sardina austral, Sprattus fuegensis, ha sido recientemente identificada en las capturas de peces pelágicos en el mar interior de Chiloé, sur de Chile. A pesar de su creciente importancia económica aún se desconocen aspectos básicos de su biología reproductiva. Se describe la actividad reproductiva de las hembras de esta especie en base a la variación mensual del Indice Gonadosomático (IGS) y a la frecuencia de estados macroscópicos de madurez ovárica entre enero de 2006 y diciembre de 2009 y de estados microscópicos a partir de febrero de 2007. Se estudia además el proceso de maduración en longitud corporal de las hembras durante la época principal de desove. Cada año, la variación mensual del IGS así como la frecuencia de estados macroscópicos de madurez, revelaron valores máximos entre septiembre y octubre, y mínimos entre enero y junio. El análisis histológico del ovario confirmó que S. fuegensis corresponde a un desovante parcial, cuya actividad reproductiva aunque tiende a concentrarse entre septiembre y octubre, puede extenderse hasta diciembre. La longitud promedio de las hembras maduras durante la estación reproductiva se estimó en 13,5cm, representando una avanzada longitud de madurez en comparación con otras estimaciones para la especie obtenidas en otras localidades. Se discute que el aumento de la actividad reproductiva en primavera, así como la maduración a mayor tamaño corporal, podrían ser parte de la estrategia reproductiva de la especie, orientada a favorecer la sobrevivencia de sus primeros estadios de vida en aguas del sur de Chile.

Palabras clave: Reproducción, época de desove, estrategia reproductiva, peces pelágicos

\section{INTRODUCCIÓN}

La sardina austral, Sprattus fuegensis Jenyns, 1842, fue reconocida hasta mediados de la década de los 80 's, con una distribución restringida solo a la costa Argentina del Atlántico sur occidental e identificada con los nombres comunes de sardina fueguina, patagonian sprat o fuegian sprat. Su distribución en la costa Atlántica de Sudamérica fue descrita desde los $40^{\circ} \mathrm{S}$ hasta el extremo sur del continente $\left(55^{\circ} \mathrm{S}\right)$ incluyendo las Islas Malvinas (Nakamura 1986, Whitehead 1989). Pequeño (1986) incluye por primera vez a la especie como parte de los clupeideos regulares 
presentes en Chile. Akira (1987), reconoce a S. fuegensis como parte importante de la dieta de la trucha café (Salmo trutta) en el fiordo Aysén del sur de Chile (5420'S$73^{\circ} 00^{\prime} \mathrm{W}$ ). Aranis et al. (2007) a través de estudios taxonómicos identifica la presencia de $S$. fuegensis en las capturas de peces de pequeño tamaño de la flota artesanal que opera en el mar interior de Chiloé, catalogándola como una especie distinta de sardina común (Strangomera bentincki). Recientemente, Bustos et al. (2008) confirman la presencia de huevos y larvas de sardina austral en el mar interior de Chiloé.

A pesar de la creciente importancia económica de Sprattus fuegensis en la zona sur de Chile, aún no existen estudios formales sobre la biología de esta especie, desconociendo aspectos básicos de su ciclo de vida como la reproducción. Al respecto, la información se restringe a los antecedentes recopilados para la especie en la costa Argentina. Estos señalan, que $S$. fuengensis corresponde a un desovante parcial con una estación de desove concentrada entre septiembre y diciembre alrededor de las Islas Malvinas (Shirakova 1978) y entre octubre y enero sobre la plataforma continental (Sánchez et al. 1995). Por otro lado, Feodorov \& Baranov (1976) y Hansen (1999), indican que las hembras tendrían una longitud media de madurez entre 11 y $12 \mathrm{~cm}$ al momento de desovar en el area de las Islas Malvinas y en la zona sur austral de la costa Argentina.

En relación al proceso reproductivo de los peces pelágicos menores, es bien sabido que el patrón estacional en los ciclos de producción de huevos de sardinas y anchovetas en zonas costeras, serían consecuencia de una sincronía de la reproducción con los ciclos ambientales de producción biológica, para favorecer la sobrevivencia de los primeros estadios de desarrollo (Cushing 1975, Bakun \& Parrish 1982, Cury \& Roy 1989, Cole \& McGlade 1998). Por ejemplo en Chile centro sur, Cubillos et al. (1999), señalaron que el periodo reproductivo de sardina común (Strangomera benticki) y anchoveta (Engraulis ringens), concentrado a principios de primavera entre los $36^{\circ}$ y $40^{\circ} \mathrm{S}$, estaría asociado, entre otros factores, a la mayor productividad de la columna de agua debido al inicio del periodo de surgencia. Durante este periodo, los estadios larvales tendrían mayor disponibilidad de alimento necesario para asegurar el crecimiento y la sobrevivencia (Cushing 1975, 1990).

En la zona sur de Chile, Iriarte et al. (2007) observaron que las mayores concentraciones de clorofila- $a$ en las aguas interiores del mar de Chiloé $\left(41^{\circ} 5^{\prime} \mathrm{S}-43^{\circ} \mathrm{S}\right)$ ocurrían durante la primavera y verano australes y mediciones in-situ indican que la mayor biomasa autotrófica ocurriría a fines de invierno y principios de primavera, así como al comienzo del otoño.
Planteamos entonces que la actividad reproductiva de Sprattus fuegensis en el mar interior de Chiloé, podría estar relacionada con estos ciclos y por lo tanto incrementar durante la primavera y/o verano australes, cuando mejoran las condiciones de alimentación y crecimiento para los estados tempranos y juveniles de la especie.

En el presente trabajo se analiza la estacionalidad de la actividad reproductiva de las hembras de Sprattus fuegensis en el mar interior de Chiloé, en base a la variación mensual o intra-anual de indicadores macro y microscopicos de la actividad gonadal, y se estudia la maduración individual de las hembras a través de la longitud durante los meses de mayor actividad reproductiva.

\section{MATeRiales y MÉTODOS}

\section{ÁREA Y PERIODO DE ESTUDIO}

El área de estudio comprendió la zona del mar interior de la isla de Chiloé, entre los $41^{\circ} 30^{\prime}$ y $43^{\circ} 30^{\prime}$ 'S (Fig. 1). La información para desarrollar este estudio se obtuvo del monitoreo biológico de sardina austral que realizó el Instituto de Fomento Pesquero de Chile (IFOP) en el marco de las pescas de investigación de la especie. El diseño de muestreo correspondió al tipo estratificado simple semanal entre enero de 2006 y diciembre de 2009.

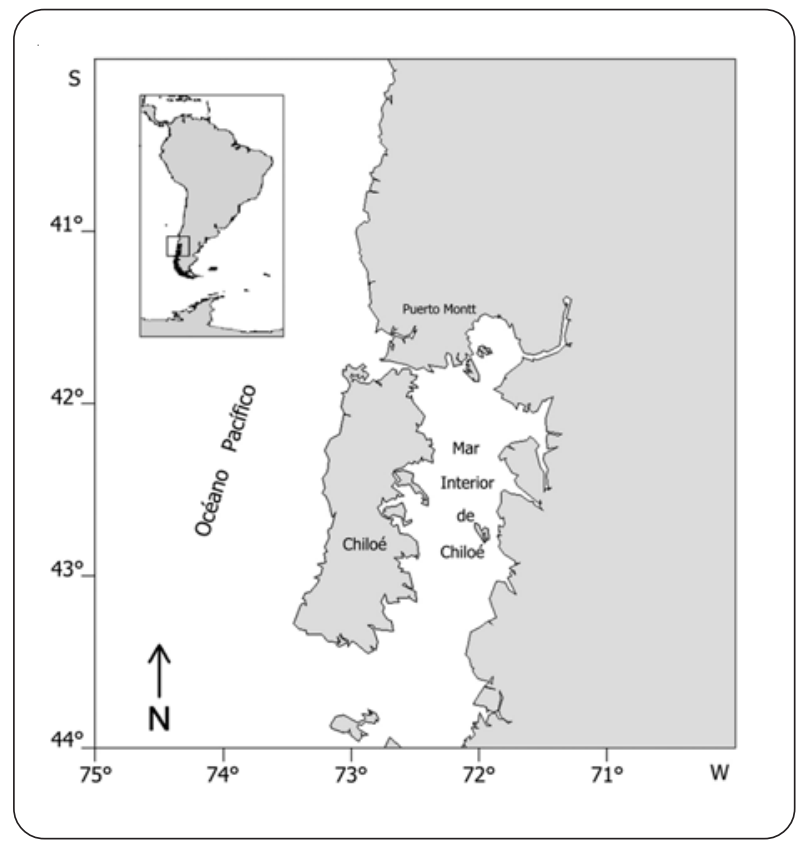

Figura 1. Área de estudio. Pesquería de pelágicos pequeños en aguas interiores del Mar del Chiloé / Study area. Small pelagic fishery in the inland waters of Chiloé 


\section{Análisis de las MUESTRAS}

El estudio consideró solamente a las hembras de Sprattus fuegensis, dado que los cambios más notables y fáciles de percibir en la actividad reproductiva de los peces, ocurre a nivel de los ovarios (Oyarzun et al. 1998). Además, las hembras representan las unidades reproductivas de las cuales depende la renovación y mantención de la población (Palumbi 2005). De esta manera, luego de identificar el sexo, las hembras fueron seleccionadas y medidas, registrando su peso $( \pm 0,1 \mathrm{~g})$ y longitud total $(\mathrm{LT} \pm 0,5 \mathrm{~cm})$. El ovario de cada ejemplar fue extraído, pesado $( \pm 0,1 \mathrm{~g})$ y calificado inicialmente dentro de una escala macroscópica de madurez (EMM) ovárica que considera cinco estados de desarrollo: virginal, incipiente, avanzado, hidratado y desovado (Holden \& Raitt 1975). Se analizó un total de 15.820 hembras de sardina austral durante todo el periodo de estudio.

A partir de febrero de 2007, se seleccionó una muestra aproximada de 50 hembras mensuales para corroborar su estado de madurez a través de criterios morfo-histólogicos. De las 15.820 hembras totales medidas, se utilizó un total de 1.534 para la observación microscópica. Para la obtención de los cortes histológicos, los ovarios fueron primero fijados en formalina neutralizada y diluida en agua de mar al 10\%. Luego, siguiendo la técnica recomendada por López et al. (1982) las muestras fueron deshidratadas en alcohol, incluidas en parafina sólida, cortadas a un espesor de $5 \mu \mathrm{m}$ y finalmente coloreadas con Hematoxilina y Eosina. Los diferentes estados de madurez, fueron establecidos según la escala microscópica de madurez ovárica modificada de Hunter \& Macewicz (1985). Las hembras fueron clasificadas dentro de siete estados de desarrollo: inmaduro (I), parcialmente vitelado (II), vitelado (III), próximo a la hidratación (IV), hidratado (V), desovado (VI) y en reposo (VII). Para el estudio de la actividad reproductiva mensual, las hembras fueron clasificadas de inactivas o activas, definiendo como inactivas aquellas hembras en estado inmaduro (I) y en reposo (VII).

\section{ÍNDICES REPRODUCTIVOS}

La actividad reproductiva de sardina austral, se describió sobre la base de la variación mensual de los índices macroscópicos (índice gonadosomático (IGS), frecuencia de estados maduros) y microscópicos (frecuencia de hembras activas, frecuencia de estados que indican desove: IV, V, VI).

El IGS se estimó a partir de una modificación de Nikolsky (1963), según la ecuación:

$$
\mathrm{IGSS}_{\mathrm{h}}=\frac{\sum \mathrm{Wg}_{\mathrm{h}}}{\sum \mathrm{W}_{\mathrm{h}}} 100
$$

donde, $\mathrm{IGS}_{\mathrm{h}}$ es el promedio dentro de cada mes (h), Wg, es el peso del ovario (g), W corresponde al peso eviscerado de las hembras.

\section{LONGITUD DE MADUREZ}

El análisis de la actividad reproductiva en relación a la longitud, consideró un total 546 hembras muestreadas durante el periodo de mayor actividad reproductiva (agostodiciembre) de los años 2008 y 2009. Se descartó el año 2007 debido al bajo número de ejemplares $(\mathrm{n}<10)$ obtenidos en los extremos de la distribución de tamaños en este periodo. Se utilizó para el análisis la escala microscópica de madurez, siendo las hembras catalogadas como maduras a partir del estado parcialmente vitelado (II) en adelante, incluyendo al estado en reposo (VII).

Un modelo del tipo logístico fue utilizado para describir la proporción de hembras maduras a la longitud (L), cuya forma está definida por la siguiente ecuación:

$$
\mathrm{P}_{\mathrm{L}}=\frac{1}{1+\exp \left(\beta_{0}-\beta_{1} * \mathrm{~L}\right)}
$$

donde, $\mathrm{P}_{\mathrm{L}}$ es la proporción de hembras maduras a la longitud (L), y $\beta_{0}$ y $\beta_{1}$, son los parámetros que representan la posición y la pendiente de la curva logística respectivamente.

Estos parámetros fueron obtenidos por Máxima Verosimilitud asumiendo una distribución binomial (maduro/inmaduro) de la variable aleatoria. La función de estimación log-verosímil tuvo la forma:

$$
\mathrm{L}\left(\beta_{0^{\prime}} \beta_{1}\right)=\sum \mathrm{k} \ln (\mathrm{P})+(1-\mathrm{h}) \ln (1-\mathrm{P})
$$

donde, $\mathrm{k}$ indica la presencia o ausencia de individuos maduros, y P es la función logística previamente descrita.

La longitud media de madurez, queda definida cuando la función logística alcanza el 50\% de probabilidad de observar individuos maduros $\left(\mathrm{L}_{50}\right)$ y se obtiene como la razón entre los parámetros $\beta_{0} \mathrm{y} \beta_{1}$, i.e.,

$$
\mathrm{L}_{50}=\frac{\beta_{0}}{\beta_{1}}
$$

donde $\mathrm{L}_{50}$, corresponde a la longitud media de madurez. El intervalo de confianza para la longitud media de madurez, 
se obtuvo mediante un enfoque de remuestreo tipo Monte Carlo de los parámetros $\beta_{0}$ y $\beta_{1}$ según lo sugerido por Roa et al. (1999) para este tipo de análisis.

\section{Resultados}

\section{ÍNDICES REPRODUCTIVOS}

Todos los indicadores analizados revelaron un incremento de la actividad reproductiva de las hembras de sardina austral durante los meses de primavera. Sin embargo, en el año 2009 se observó una extensión del periodo reproductivo encontrando valores altos de los índices cualitativos de madurez hacia fin de año.

El índice gonadosomático (IGS) promedio reveló valores altos en los meses de septiembre y octubre de cada año, un descenso evidente a partir de noviembre y valores mínimos entre diciembre y julio (Fig. 2). De la misma forma, aunque la presencia de ovarios con algún grado de madurez se observó a lo largo de todo el año, el análisis macroscópico de la gónada indicó una mayor frecuencia de ovarios en estado de madurez avanzada entre septiembre y octubre. Por su parte, las hembras en evidente actividad reproductiva (ovarios hidratados), se observaron cada año, principalmente entre septiembre y noviembre (Fig. 3). Durante el año 2009, las hembras hidratadas se observaron durante todo el segundo semestre, excepto en octubre donde la veda implementada este año, impidió obtener información. En enero de 2008 y enero-febrero de 2009 no se registró información para esta variable debido a que no hubo operación de pesca.

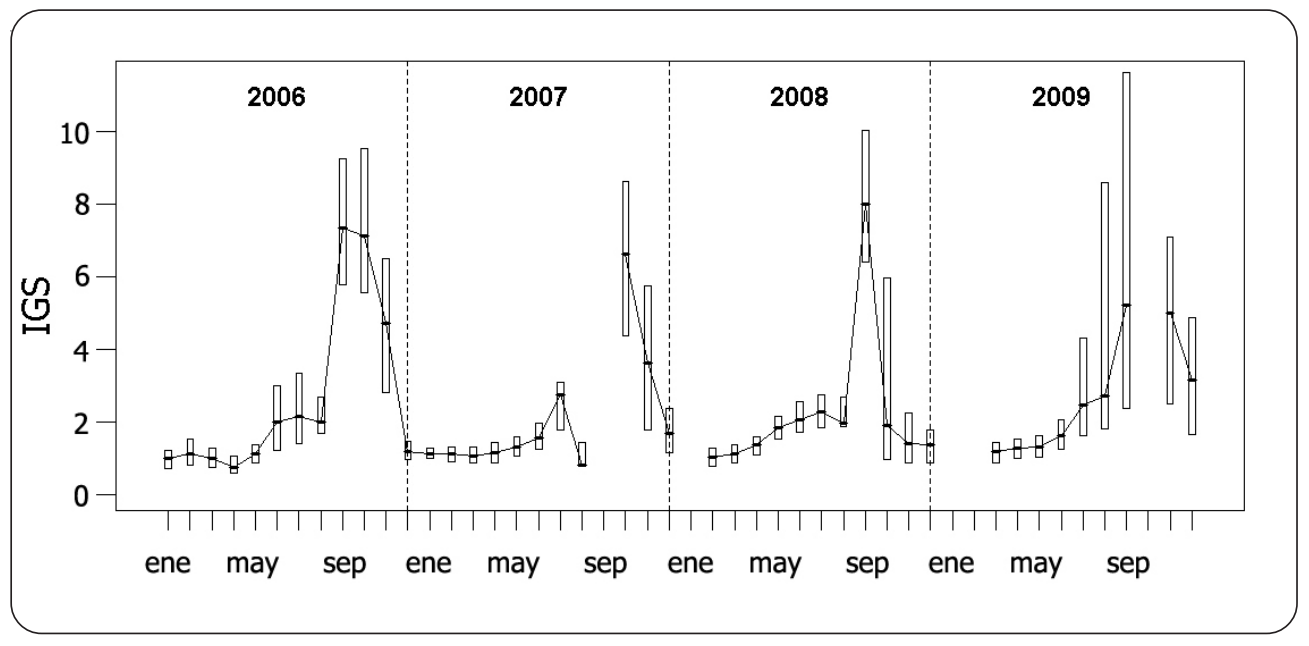

Figura 2. Variación mensual del índice gonadosomático promedio de las hembras de Sprattus fuegensis en las aguas interiores de Chiloé / Monthly variations of the gonadosomatic index for females of Sprattus fuegensis in the inland waters of Chiloé

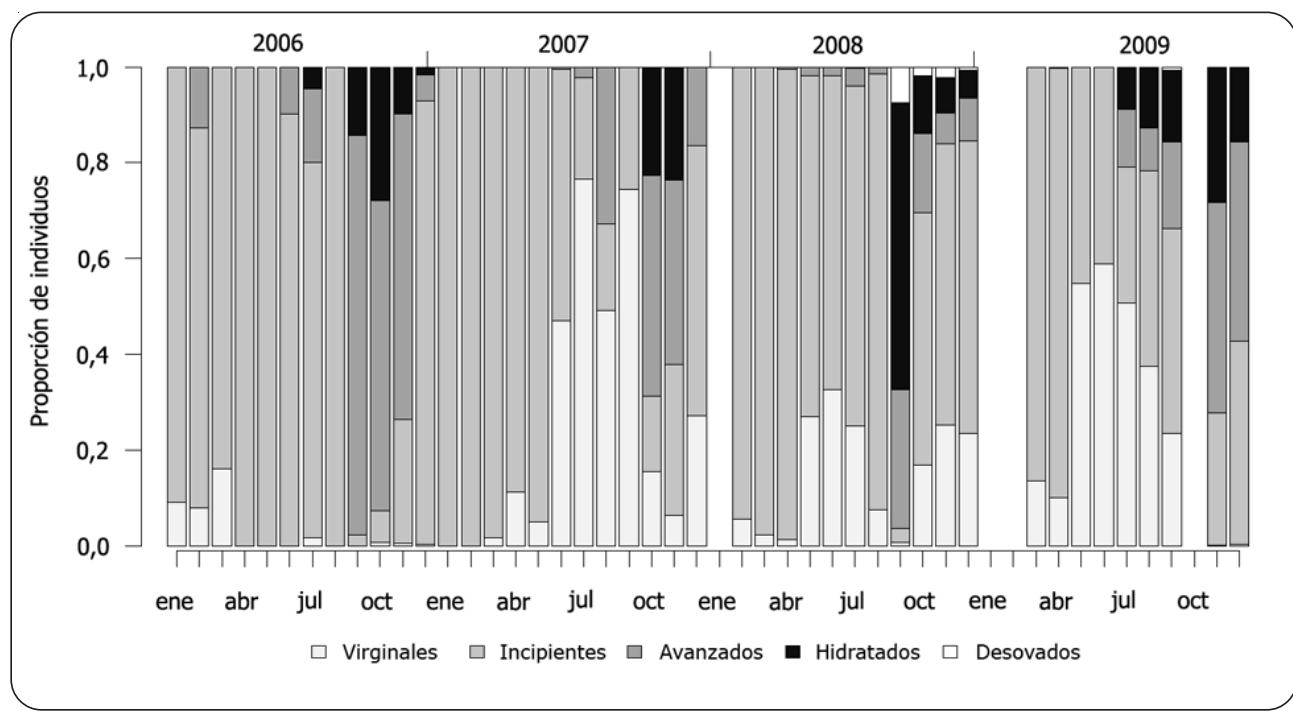

Figura 3. Proporción mensual de los estados de madurez (EM) macroscópicos de Sprattus fuegensis en las aguas interiores de Chiloé / Monthly proportion of macroscopic maturity stages (EM) of Sprattus fuegensis in the inland waters of Chiloé 
El análisis histológico del ovario reveló un desarrollo de tipo asincrónico, con presencia de ovocitos en distintos estados de madurez dentro de un mismo ovario (Fig. 4). Los resultados de este análisis además corroboran, que la actividad reproductiva de la especie se desarrolla casi exclusivamente durante el segundo semestre de cada año, excepto el año 2007, donde también se observó una pequeña fracción de individuos activos en febrero. Durante el año 2009, la presencia de ovarios con ovocitos activos se apreció, aunque en una baja proporción, a partir de mayo, y la proporción más alta, en diciembre (Fig. 5). Las hembras en manifiesto proceso de desove, es decir con ovarios en estados próximos a la hidratación, hidratados y desovados, se apreciaron a partir de agosto en el 2007 y de septiembre en el 2008, mostrando la mayor proporción en octubre y septiembre, respectivamente. Los meses sin información, se debió a la escasa calidad de las muestras para el análisis histológico. En el año 2009 la falta de muestras histológicas en septiembre y octubre impidió corroborar el desove durante estos meses, sin embargo, fue posible apreciar una alta proporción de estados próximos a la hidratación y desovados en noviembre y diciembre (Tabla 1 ).

Tabla 1. Número de hembras por estado de madurez que indican desove: próximo a la hidratación (IV), hidratadas (V) y desovadas (VI). Número de hembras totales, numero y porcentaje de hembras en proceso de desove entre enero de 2007 y diciembre de 2009. Los datos entre enero y julio fueron agrupados para cada año, debido a la escasa presencia de estados indicadores de desove en este periodo / Number of females in maturity state indicating spawning: nearly hydrated (IV), hydrated (V) and spawned. (VI). Total number of females monthly analyzed, number and percentage of them in spawning process between J anuary 2007 and December 2009. Data from J anuary and J uly were pooled for each year due to the scarcity spawning state indicators in this time

\begin{tabular}{|c|c|c|c|c|c|c|}
\hline \multirow{2}{*}{ Año } & \multirow{2}{*}{ Meses } & \multicolumn{3}{|c|}{ Estado de madurez } & \multirow{2}{*}{$\begin{array}{c}\text { Hembras } \\
\text { totales }\end{array}$} & \multirow{2}{*}{$\begin{array}{c}\text { En proces } \\
\text { desove }\end{array}$} \\
\hline & & IV & $\mathrm{V}$ & VI & & \\
\hline \multirow[t]{6}{*}{2007} & Ene-Jul & 0 & 0 & 2 & 239 & $2(0,8 \%)$ \\
\hline & Ago & 19 & 5 & 4 & 50 & $28(56 \%)$ \\
\hline & Sep & $=$ & - & - & - & - \\
\hline & Oct & 7 & 3 & 32 & 50 & $42(84 \%)$ \\
\hline & Nov & - & - & - & - & - \\
\hline & Dic & 2 & 0 & 14 & 41 & $16(39 \%)$ \\
\hline \multirow[t]{6}{*}{2008} & Ene-Jul & 0 & 0 & 1 & 266 & $1(0,4 \%)$ \\
\hline & Ago & 0 & 0 & 0 & 58 & $0(0,0 \%)$ \\
\hline & Sep & 39 & 1 & 38 & 85 & $78(92 \%)$ \\
\hline & Oct & - & - & - & - & \\
\hline & Nov & 6 & 0 & 7 & 129 & $13(10 \%)$ \\
\hline & Dic & 8 & 0 & 1 & 89 & $9(10 \%)$ \\
\hline \multirow[t]{6}{*}{2009} & Ene-Jul & 0 & 0 & 1 & 342 & $1(0,3 \%)$ \\
\hline & Ago & 4 & 0 & 0 & 65 & $4(6,2 \%)$ \\
\hline & Sep & - & - & - & - & - \\
\hline & Oct & - & - & - & - & - \\
\hline & Nov & 17 & 2 & 15 & 58 & $34(59 \%)$ \\
\hline & Dic & 17 & 8 & 32 & 62 & $57(92 \%)$ \\
\hline
\end{tabular}

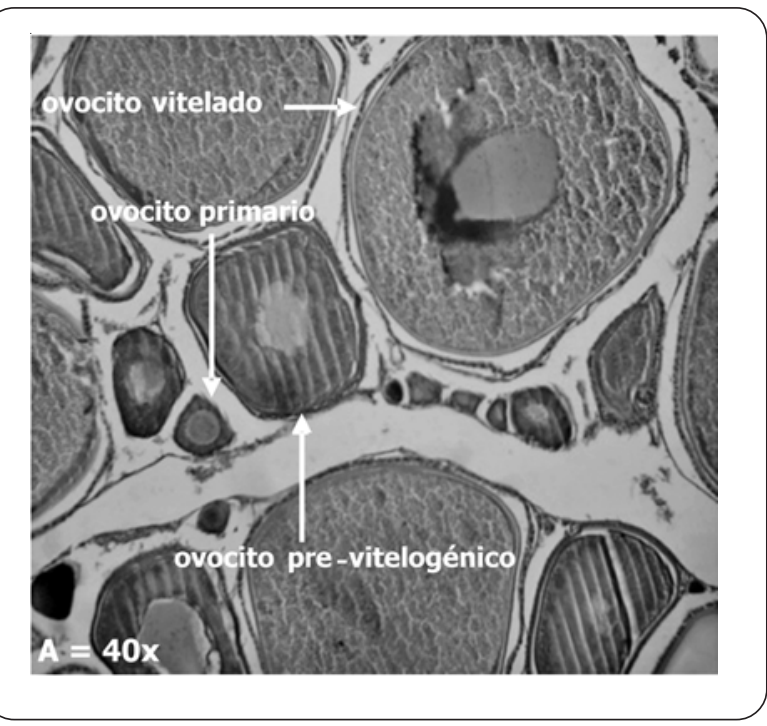

Figura 4. Fotografía de preparación histológica de ovario de Sprattus fuegensis con ovocitos en diferentes estados de desarrollo. La muestra corresponde a una hembra obtenida durante noviembre de 2009 / Photography of histological ovary preparation of Sprattus fuegensis with oocytes in different stages of development. The sample corresponds to a female collected during November 2009

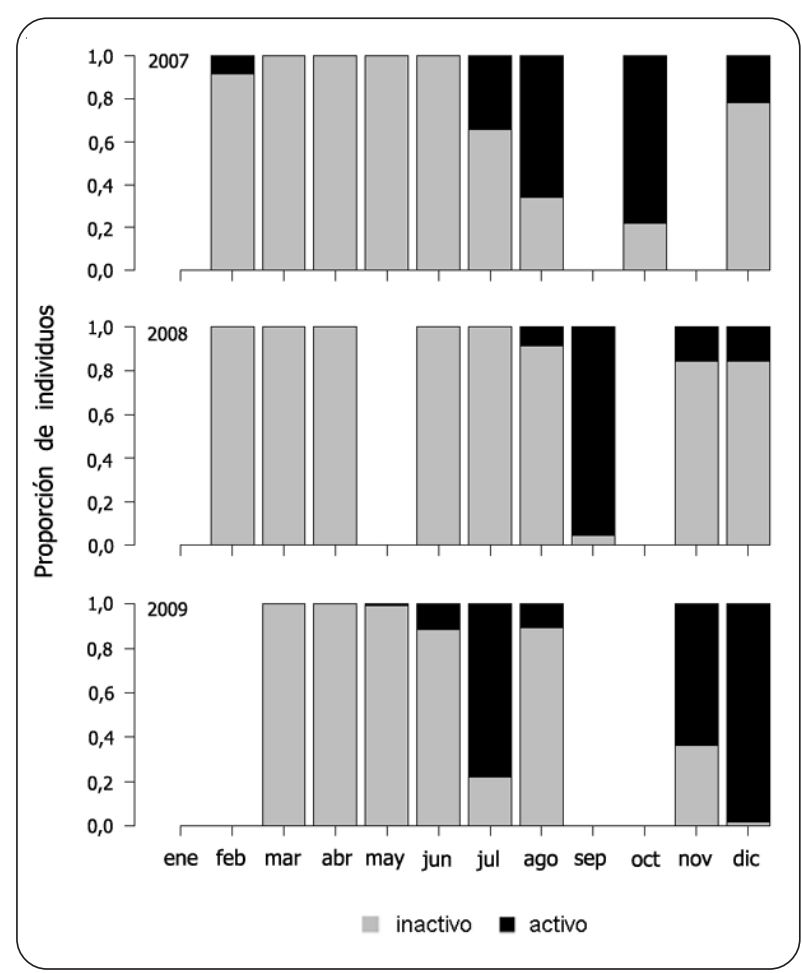

Figura 5. Proporción mensual de ovarios sexualmente activos de Sprattus fuegensis en las aguas interiores de Chiloé clasificados a través de la técnica histológica / Monthly proportion of sexually active ovaries of Sprattus fuegensis in the inland waters of Chiloé classified by the histological technique 


\section{LONGITUD DE MADUREZ}

Los resultados del modelo logístico (Fig. 6) indican que bajo los $11,5 \mathrm{~cm}$, la probabilidad de observar hembras maduras es baja, y sobre los $15 \mathrm{~cm}$ existe una alta probabilidad que la mayoría de las hembras se encuentren maduras. La longitud media de madurez $\left(\mathrm{L}_{50}\right)$ de $S$. fuegensis entre agosto y diciembre, se estimó en 13,52 cm con un intervalo de confianza al 95\% entre 13,39 y 13,71 cm de LT. Los parámetros $\beta_{0}$ y $\beta_{1}$ que describen la ecuación logística, alcanzaron valores de 25,87 y -1,91 respectivamente, con error estándar de 2,61 para $\beta_{0}$ y de 0,20 para $\beta_{1}$.

\section{Discusión}

El período principal de desove de sardina austral descrito en este estudio en septiembre y octubre coincide parcialmente con lo informado para la especie por Shirakova (1978) y Sánchez et al. (1995), quienes reportaron una época principal de reproducción para $S$. fuegensis que se extiende entre septiembre y diciembre alrededor de las Islas Malvinas y desde octubre a enero en la costa Argentina, respectivamente.

Los altos valores de IGS en septiembre y octubre, así como la mayor frecuencia de ovarios macroscópicamente maduros (EMM), concuerdan con el desove principal de fines de invierno y principios de primavera reportado para otros peces pelágicos pequeños como la sardina común y anchoveta en la costa de Chile (Cubillos \& Arancibia 1993, Cubillos 1999, Canales \& Leal 2009).

El análisis histológico del ovario confirmó que Sprattus fuegensis corresponde a un desovante parcial, lo que había sido indicado por otros autores para esta especie en la costa Argentina (Hansen 1999). La presencia simultánea de ovocitos en distintos estados de desarrollo, demuestra, de acuerdo a Wallace \& Selman (1981), que sardina austral puede llevar a cabo múltiples puestas durante el ciclo reproductivo anual. Su ovario de desarrollo asincrónico, evidencia un tipo de reproducción común con los otros clupeideos presentes en Chile (Cubillos et al. 1999) y con un período prolongado de actividad reproductiva durante el segundo semestre de cada año, que incrementa durante los meses de primavera.

Diferentes hipótesis han sido planteadas para explicar el patrón estacional observado en el ciclo reproductivo de los peces pelágicos menores. Procesos relacionados a la estabilidad de la columna de agua (Lasker 1975), producción secundaria para la alimentación larval (Cushing 1975, 1990), turbulencia y transporte (Bakun \& Parrish 1982, Parrish et al. 1983) y velocidades optimas de viento (Cury \& Roy 1989), han sido señalados como importantes factores

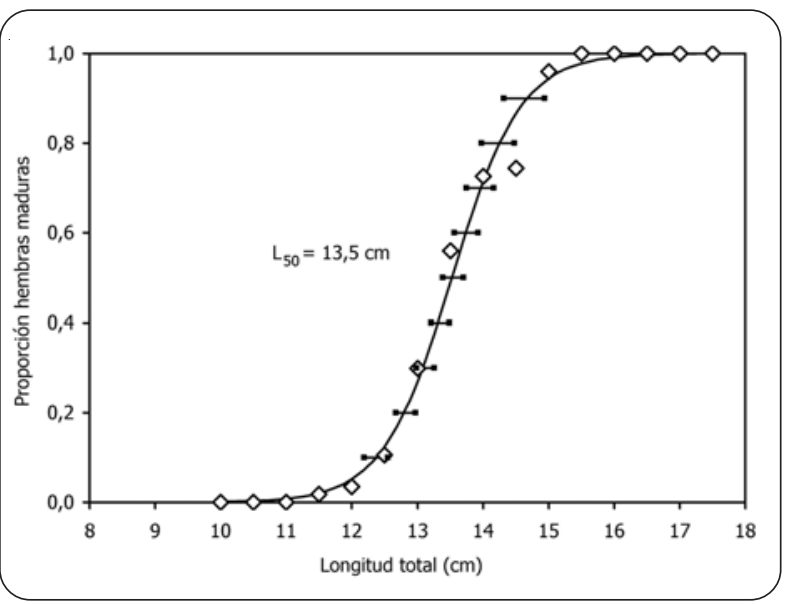

Figura 6. Ojiva de madurez de Sprattus fuegensis en las aguas interiores de Chiloé / Maturity ogive of Sprattus fuegensis in the inland waters of Chiloé

que determinan los periodos y áreas donde ocurre el desove. En la costa de Chile, Yáñez et al. (1992), Cubillos et al. (1999, 2001) y Cubillos \& Arcos (2002) también describen un efecto significativo del ambiente sobre los procesos reproductivos de sardinas y anchovetas.

En el presente estudio, la regularidad interanual en los índices reproductivos de sardina austral, confirman el planteamiento inicial en relación a que la actividad reproductiva de la especie debería incrementar en los meses de primavera, cuando, la producción biológica y la temperatura en el mar interior de Chiloé también aumentan (Iriarte et al. 2007). Durante este periodo, los primeros estadíos de desarrollo deberían encontrar mejores condiciones para la alimentación y crecimiento. De la misma forma, la extensión en el periodo de desove durante el año 2009, sugerida por la presencia de ovocitos activos a partir de mayo y la alta proporción presente hasta diciembre, podría estar relacionada con algún tipo de variación temporal en las condiciones ambientales presentes en el área de estudio.

En cuanto al proceso de maduración en función del tamaño corporal, la longitud media de las hembras maduras durante el periodo de desove, fue superior a la informada para Sprattus fuegensis en la costa Argentina. Feodorov \& Baranov (1976) y Hansen (1999) coinciden en señalar que esta especie alcanzaría una longitud de $50 \%$ de madurez entre los 11 y $12 \mathrm{~cm}$, correspondiente a una edad de entre 1,72 y 2,05 años. De igual manera, la ojiva de madurez aquí descrita, se encuentra desplazada hacia longitudes superiores en comparación con las estimaciones realizadas sobre otro representante de la familia, en la zona costera de Chile central (36-40º). Según Cubillos et al. (1999), Strangomera benticki (sardina común) alcanza la longitud media de 
madurez entre los 10 y $11 \mathrm{~cm}$, al final del primer año de vida. De acuerdo, a los parámetros de crecimiento reportados por Cerna (2005), sardina austral alcanzaría la $\mathrm{L}_{50}$ de 13,5 cm en torno al año y medio de vida y el 100\% de individuos maduros a los $15 \mathrm{~cm}$, al final del segundo año.

Un importante parámetro reproductivo asociado a la longitud corporal, es la fecundidad. Las hembras maduras de mayor tamaño son capaces de liberar un mayor número de huevos al ambiente (Hunter \& Goldberg 1980, Morita \& Takashima 1990, Arancibia et al. 1994). También, se ha comprobado en diferentes grupos de peces que las hembras de mayor tamaño o edad producen huevos más grandes, con mayor cantidad de vitelo para el desarrollo y crecimiento de la larva (Clarke 1989, Chambers et al. 1989, Berkeley et al. 2004, Palumbi 2005). En sardina austral, Landaeta et al. (en prensa) realizando mediciones de sus huevos planctónicos en la zona de los canales del sur de Chile, describen mayores diámetros promedios (0,92-1,36 mm) y en un rango más amplio que lo informado previamente por Ciechomski (1971) (1,07-1,03 mm) para la especie en la costa Argentina y por Herrera et al. (1987) (0,89-1,03 mm) para la otra especie de sardina (Strangomera benticki) en Chile central. De esta manera, es posible sugerir, que el desove a longitudes avanzadas en el mar interior de Chiloé, podría también formar parte de la estrategia reproductiva de la especie destinada a incrementar su fecundidad y/o producir huevos de mejor calidad, lo que favorecería una mayor sobrevivencia de larvas y juveniles.

A partir de los resultados de este estudio, se concluye que la actividad reproductiva de Sprattus fuegensis en el mar interior de Chiloé, incrementa durante el segundo semestre del año y aunque el desove tiende a concentrarse entre septiembre y octubre, este puede extenderse hasta el mes de diciembre. Se estableció además que la especie corresponde a un desovante parcial, y que las hembras tenderían a desovar tardíamente a una longitud promedio de 13,5 cm durante la época principal de desove. Se postula que tanto la época principal de desove como la maduración de las hembras a longitudes avanzadas, podrían ser parte de la estrategia reproductiva de la especie, orientada a favorecer la sobrevivencia de sus estadios tempranos de vida bajo las condiciones ambientales presentes en la zona del mar interior de Chiloé.

Si bien este trabajo constituyó una primera aproximación en el conocimiento de la dinámica reproductiva de la especie en Chile y de su ciclo de vida, el estudio de otros parámetros asociados a la reproducción como la fecundidad, tamaño y calidad del huevo, así como el efecto de factores endógenos y exógenos sobre actividad ovárica y tasas de desarrollo, son necesarios para mejorar la comprensión de la dinámica reproductiva de sardina austral y su relación con la variabilidad ambiental.

\section{Agradecimientos}

Los autores agradecen a la Subsecretaría de Pesca de Chile y las asociaciones de pescadores artesanales de la X Región por la disponibilidad de la información para el estudio. Especial reconocimiento al esfuerzo del personal del Instituto de Fomento Pesquero (IFOP) que colecta las muestras en terreno. Se agradecen además los valiosos comentarios de los revisores anónimos de este trabajo.

\section{LITERATURA CITADA}

Akira Z. 1987. Biological observation of sea-run brown trout in fiordo Aysen, sourthen Chile (Pisces: Salmonidae). Revista de Biología Marina 23(2): 193-213.

Arancibia H, L Cubillos, J Remmaggi \& R Alarcón. 1994. Determinación de la talla de primera madurez sexual y fecundidad parcial en la sardina común, Strangomera bentincki (Norman, 1936), del área de Talcahuano, Chile. Biología Pesquera 23: 11-17.

Aranis A, R Meléndez, G Pequeño \& F Cerna. 2007. Sprattus fuegensis en aguas interiores de Chiloé, Chile (Osteichthyes: Clupeiformes: Clupeidae). Gayana 71(1): 102-113.

Bakun A \& R Parrish. 1982. Turbulence, transport, and pelagic fish in the California and Peru Current systems. CALCOFI Reports 123: 99-112.

Berkeley S, C Chapman \& S Sogard. 2004. Maternal age as a determinant of larval growth and survival in a marine fish, Sebastes melanops. Ecology 85(5): 1258-1264.

Bustos C, M Landaeta \& F Balbontín. 2008. Efectos ambientales sobre la variabilidad espacial del ictioplancton de Chile austral durante noviembre de 2005. Revista Chilena de Historia Natural 81: 205-219.

Canales TM \& E Leal. 2009. Parámetros de historia de vida de la anchoveta (Engraulis ringens Jenyns, 1842) distribuida en la zona centro norte de Chile. Revista de Biología Marina y Oceanografía 44(1): 173-179.

Cerna F. 2005. Estimación de la edad de sardina austral a través de otolitos. En: Aranis A, L Caballero, F Cerna, A Gomez, A Lopez \& C Bernal (eds). Monitoreo de la pesquería de pequeños pelágicos en aguas interiores de la X Región, año 2004. Informe Final. Proyecto FIP N²00439: 1-96.

Chambers R, C Leggett \& J Brown. 1989. Egg size, female effects, and the correlations between early life history traits: an appraisal at the individual level. Fishery Bulletin 87: 515-523. 
Ciechomski J. 1971. Estudio sobre los huevos y larvas de la sardine fueguina Sprattus fuegensis y de Maurolicus muelleri, hallados en aguas adyacentes al sector patagónico Argentino. Physis 30: 557-567.

Clarke TA. 1989. Seasonal differences in spawning, egg size, and early development time of the hawaiian anchovy or nehu, Encrasicholina purpurea. Fishery Bulletin 87(3): 593-600.

Cole J \& J McGlade. 1998. Clupeoid population variability, the environment and satellite imagery in coastal upwelling systems. Reviews in Fish Biology and Fisheries 8: 445471.

Cubillos L \& H Arancibia. 1993. Análisis de la pesquería de sardina común (Strangomera bentincki) y anchoveta (Engraulis ringens) del área de Talcahuano, Chile. Investigaciones Marinas 21: 3-21.

Cubillos L \& D Arcos. 2002. Recruitment of common sardine (Strangomera bentincki) and anchovy (Engraulis ringens) off central-south Chile in the 1990s and the impact of the 1997-1998 El Niño. Aquatic Living Resources 15: 87-94.

Cubillos L, TM Canales, D Bucarey, A Rojas \& R Alarcón. 1999. Época reproductiva y talla media de primera madurez de Strangomera bentincki, y Engraulis ringens en el período 1993-1997 en la zona centro sur de Chile. Investigaciones Marinas 27: 73-85.

Cubillos L, D Arcos, M Canales \& D Bucarey. 2001. Seasonal growth of small pelagic fish off Talcahuano $\left(37^{\circ} \mathrm{S}-73^{\circ} \mathrm{W}\right)$, Chile: a consequence of their reproductive strategy to seasonal upwelling? Aquatic Living Resource 14: $115-124$.

Cury P \& C Roy. 1989. Optimal environmental window and pelagic fish recruitment success in upwelling areas. Canadian Journal of Fisheries and Aquatic Sciences 46: 670-680.

Cushing DH. 1975. Marine ecology and fisheries, 275 pp. Cambridge University Press. Cambridge.

Cushing DH. 1990. Plankton production and yearclass strength in fish populations: an update of the match/ mismatch hypothesis. Advances in Marine Biology 26: 249293.

Feodorov S \& A Baranov. 1976. Algunos datos nuevos acerca la biología de sardina fueguina. Hoz-va I Okeanografii Kaliningrad 65: 135-142. [Original en ruso]

Hansen JE. 1999. Estimación de parámetros poblacionales del efectivo de sardina fueguina (Spratus fuegensis) de la costa continental argentina. INIDEP. Informe Técnico 27: $1-18$.

Herrera G, E Tarifeño \& M Orellana. 1987. Descripción de huevos y primeras fases larvales de sardina común (Strangomera benticki) y del machuelo (Ethmidum maculatum). Biología Pesquera 16: 107-113.
Holden M \& D Raitt. 1975. Manual de ciencia pesquera. Parte 2. Métodos para investigar los recursos y su aplicación. FAO Documento Técnico de Pesca 115: 1-211.

Hunter J \& S Goldberg. 1980. Spawning incidence and batch fecundity in northern anchovy, Engraulis mordax. Fishery Bulletin 77: 641-652.

Hunter J \& B Macewicz. 1985. Rates of atresia in the ovary of captative and wild northern anchovy, Engraulis mordax. Fishery Bulletin 83: 119-136.

Iriarte J, H Gonzáles, K Liu \& C Valenzuela. 2007. Spatial and temporal variability of chlorophyll and primary productivity in surface waters of southern Chile (41.5-43 ${ }^{\circ}$ S). Estuarine, Coastal and Shelf Science 74: 471-480.

Landaeta MF, CA Bustos, P Palacios-Fuentes, P Rojas \& F Balbontín. (en prensa). Distribución del ictioplancton en la Patagonia austral de Chile: potenciales efectos del deshielo de Campos de hielo sur. Latin American Journal of Aquatic Research.

Lasker R. 1975. Field criteria for survival of anchovy larvae: the relationship between inshore chlorophyll maximum layers and successful first feeding. Fishery Bulletin 73: 453-462.

López L, C Leyton \& M Graf. 1982. Técnicas de histología y citología, 141 pp. Departamento de Biología Celular y Genética, Facultad de Medicina, Universidad de Chile, Santiago.

Morita K \& Y Takashima. 1990. Effect of female size on fecundity and egg size in white-spotted charr: comparison between sea-run and resident forms. Journal of Fish Biology 53: 1140-1142.

Nakamura I. 1986. Important fishes trawled off Patagonia, 369 pp. JAMARC, Tokyo.

Nikolsky GV. 1963. The ecology of fishes, 352 pp. Academic Press, London.

Oyarzún C, J Chong \& M Malagueño. 1998. Fenología reproductiva en jurel, Trachurus symmetricus (Ayres, 1855) (Perciformes, Carangidae) en el área de TalcahuanoChile: 1982-1984. En: Arcos D (ed). Biología y ecología del jurel en aguas chilenas, pp. 67-76. Instituto de Investigación Pesquera, Chile.

Palumbi SR. 2005. Why mothers matter. Nature. 30: 621622.

Pequeño G. 1986. Peces de Chile. Lista sistemática revisada y comentada. Revista de Biología Marina 24(2): 1-132.

Roa R, B Ernst \& F Tapia. 1999. Estimation of size at sexual maturity: an evaluation of analytical and resampling procedures. Fishery Bulletin 97: 570-580.

Sánchez R, A Remelso, A Madirolas \& J Ciechomski. 1995. Distribution and abundance of post-larvae and juveniles of the Patagonian sprat, Sprattus fuegensis, and related hydrographic conditions. Fisheries Research 23: 47-81. 
Shirakova EN. 1978. Contribution to the biology of Tierra del Fuego sprat Sprattus fuegensis (Jenyns, 1842). Biology of the Sea 3: 78-84.

Wallace R \& K Selman. 1981. Cellular and dynamic aspects of oocyte growth in teleosts. American Zoologist 21: 325343.

Whitehead PJ. 1989. Clupeoid fishes of the world. An annotated and illustrated catalogue of the herrings, sardines, pilchards, sprats, anchovies and wolfherrings. Part 1. Chirocentridae, Clupeidae and Pristigasteridae. FAO Fisheries Synopsis 125(7), Part 1: 1-303.

Yáñez E, M Barbieri \& L Santillán. 1992. Long-term environmental variability and pelagic fisheries in Talcahuano, Chile. En: Payne A, L Brink, K Mann \& R Hilborn (eds.). Benguela Trophic Functioning. South African Journal Marine Science. 12: 175-188.

Recibido el 8 de julio de 2010 y aceptado el 26 de enero de 2011 